7. Slavin D et al. Biol Reprod 1999; 61: 1586-1591.

8. Gehrau RC et al. Biochim Biophys Acta 2005; 1730: 137-146.

9. Narla G et al. Science 2001; 294: 2563-2566.

10. Reeves HL et al. Gastroenterology 2004; 126: 1090-1103.

11. Lievre A et al. Oncogene 2005; 24: 7253-7256.

12. Matsumoto $\mathrm{N}$ et al. Blood 2006; 107: 1357-1365.
13. Narla G et al. Oncogene 2007; 26: 4428-4434.

14. Sirach E et al. Cell Death Differ 2007; 14: 1202-1210.

15. Vichi $P$ et al. EMBO J 1997; 16: 7444-7456.

16. Demidenko ZN et al. Cancer Res 2004; 64: 3653-3660.

17. Slavin DA et al. Oncogene 2004; 23: 8196-8205.

18. Banck MS et al. FEBS Lett 2006; 580: 6981-6986.

\title{
Unexpected role of the phosphate carrier in mitochondrial fragmentation
}

\author{
Cell Death and Differentiation (2008) 15, 616-618; doi:10.1038/sj.cdd.4402295; published online 4 January 2008
}

Dear Editor,

Mitochondrial carriers constitute a family of hexa-transmembrane proteins that participate in the transport of metabolites across the inner mitochondrial membrane. ${ }^{1}$ Several members of this family have also been proposed to participate in the apoptotic processes. For instance, distinct isoforms of the adenine nucleotide translocase have been involved in the regulation (either positive or negative) of mitochondrial membrane permeabilization. ${ }^{2-5}$ Similarly, the mitochondrial carrier homolog 2 (Mtch2) may mediate Bcl-2-dependent apoptosis inhibition. ${ }^{6}$

Recently, we and others have found that yet another protein from the mitochondrial carrier family, the inorganic phosphate carrier $(\mathrm{PiC})$, may participate in the regulation of mitochondrial membrane permeabilization. A genome-wide cDNA screen identified $\mathrm{PiC}$ as one of the few proteins that, if overexpressed, is able to efficiently trigger the intrinsic pathway of apoptosis. ${ }^{7}$ $\mathrm{PiC}$ was also found to functionally interact with the cytomegalovirus (CMV)-encoded protein VMIA (i.e. viral mitochondrial inhibitor of apoptosis). ${ }^{8,9}$ VMIA inhibits apoptosis by recruiting Bax to mitochondria, thereby provoking its inactiva- tion. $^{10,11}$ In addition, VMIA is responsible for the early cytopathic effect associated with CMV infection, ${ }^{8}$ independently from its interaction with Bax. ${ }^{8,12}$ Moreover, vMIA inhibits the PiC-mediated transport of inorganic phosphate across the inner mitochondrial membrane, thereby reducing ATP synthesis (which is strictly dependent on phosphate import). In turn, this results in a disorganization of the actin cytoskeleton and rounding up of the cells, which characterize the CMV-mediated early cytopathic effect. ${ }^{8}$

We have recently reported that, similar to Bax, vMIA can stimulate the fragmentation of the mitochondrial network. ${ }^{13}$ This is intriguing and counterintuitive because VMIA is a strong inhibitor of apoptosis, ${ }^{9}$ while mitochondrial fragmentation is often associated with apoptosis induction. ${ }^{14}$ Since VMIA-mediated mitochondrial fragmentation occurs even in Bax-depleted cells, ${ }^{8}$ this effect must be independent of the VMIA-mediated recruitment of Bax to the outer mitochondrial membrane. We therefore sought whether the depletion of PiC with two distinct small interfering RNAs (siRNAs) (Figure 1a; Supplementary Materials and Methods) might affect the

Figure 1 (a-c) Effect of the depletion of the mitochondrial phosphate carrier $(\mathrm{PiC})$ on mitochondrial morphology and vMIA activity. (a) Efficacy of the siRNA-mediated downregulation of PiC. PiC was depleted by using two distinct siRNAs (see Supplementary Materials and Methods) in HeLa control cells (HeLa Neo) and in cells overexpressing VMIA (HeLa VMIA), and the abundance of the mRNA transcript was assessed by RT-PCR $24 \mathrm{~h}$ post-transfection, as compared to non-transfected cells $(-)$ and cells transfected with an irrelevant siRNA (UNR). (b) Impact of vMIA and PiC on mitochondrial dynamics. Forty-eight hours after siRNA transfection, cells were fixed and stained with an antibody recognizing the core 2 subunit of complex III of the respiratory chain (red fluorescence). Representative images are shown (scale bars represent $2 \mu \mathrm{m}$ ). (c) Influence of PiC on vMIA-mediated apoptosis suppression. PiC was depleted by means of two distinct siRNAs in HeLa Neo and HeLa vMIA cells. Twenty-four hours later, cells were treated for additional $6 \mathrm{~h}$ with $500 \mathrm{ng} / \mathrm{ml}$ anti-CD95 monoclonal antibody $(\alpha-C D 95)$ plus $100 \mu \mathrm{g} / \mathrm{ml}$ cycloheximide $(\mathrm{CHX})$ or, alternatively, with $500 \mathrm{nM}$ staurosporine (STS) and stained with the $\Delta \Psi_{\mathrm{m}}$-sensitive dye DiOC 6 (3) as well as the membrane-impermeant dye propidium iodide (PI) for the cytofluorometry-assisted quantification of dying and dead cells. White and black columns indicate the percentage of cells exhibiting the loss of $\Delta \Psi_{\mathrm{m}}\left(\mathrm{DiOC}_{6}(3)^{\text {low }}\right)$ alone $\left(\mathrm{PI}^{-}\right)$or together with ruptured plasma membranes $\left(\mathrm{PI}^{+}\right)$, respectively (mean $\pm \mathrm{STE} ; n=3$ ). Statistical significance was assessed by means of paired Student's $t$-tests comparing HeLa Neo and HeLa vMIA cells subjected to the same siRNA transfection and pro-apoptotic stimulation (NS = non-statistically significant). (d and e) Effect of PiC knockdown on mitochondrial fragmentation induced by the depletion of mitofusin 2 (Mfn-2). HeLa Neo cells were co-transfected with an irrelevant siRNA (UNR) or with siRNAs for the depletion of Mfn-2 and PiC in the indicated combinations for $72 \mathrm{~h}$, followed by staining with MitoTracker ${ }^{\mathbb{R}}$ Orange for the assessment of mitochondrial morphology. (d) Representative pictures (at different magnifications) of cells exhibiting an intact mitochondrial network (siRNA UNR, siRNA Mfn-2 + PiC; panels 1 and 4 respectively) or micro- versus macrofragmentation of the organelles (siRNA Mfn-2 + UNR; panels 3 and 4, respectively). Scale bars represent $2 \mu \mathrm{m}$. (e) Mitochondrial fragmentation induced by the depletion of Mfn-2 in the presence and in the absence of PiC. For each condition, the percentage of cells displaying macrofragmented mitochondria was assessed in a population of at least 300 cells (mean \pm STE; $n=3$ ). Paired Student's $t$-tests were performed to assess the statistical significance of the indicated comparisons 
fragmentation of the mitochondrial network promoted by vMIA. The transient (not shown) or stable transfection with VMIA clearly caused a major fragmentation of the mitochondrial network from a tubular to a dot-like appearance in several cell lines, including HeLa cervical carcinoma cells (Figure 1b), HCT116 colon carcinoma cells and human fibroblasts (not shown). This fragmentation was prevented by the downregulation of $\mathrm{PiC}$ by means of two distinct siRNAs (Figure 1b).
As a note, $\mathrm{PiC}$ depletion did not influence per se mitochondrial morphology (Figure 1b). In spite of this major - and unexpected - influence of $\mathrm{PiC}$ on mitochondrial dynamics, $\mathrm{PiC}$ depletion did not affect the antiapoptotic activity of VMIA. Expression of vMIA protected HeLa cells against mitochondrial dysfunction and cell death induced by staurosporine (a general kinase inhibitor that promotes the intrinsic pathway of apoptosis) or by crosslinking the death receptor CD95/Fas

a

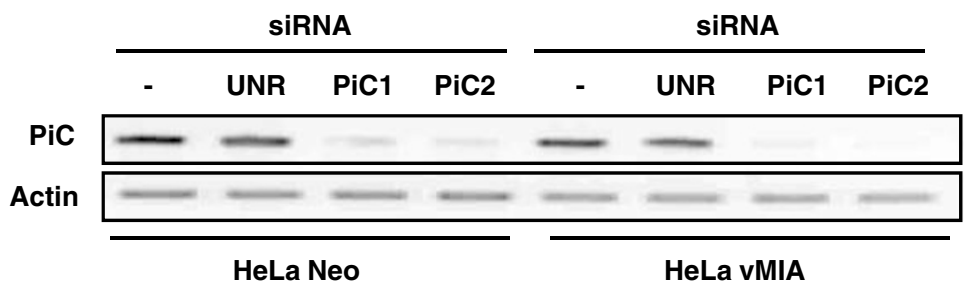

b

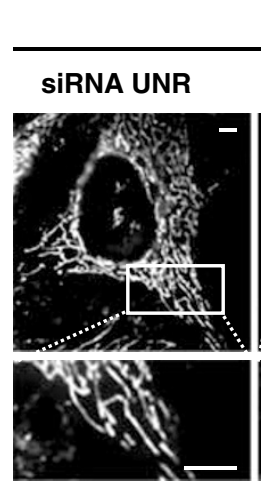

HeLa Neo SiRNA PiC1
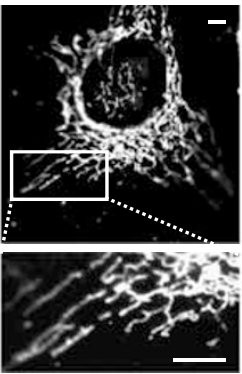
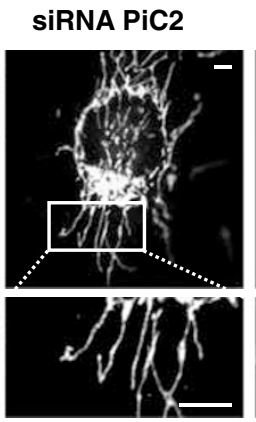

HeLa vMIA

C

c

HeLa Neo

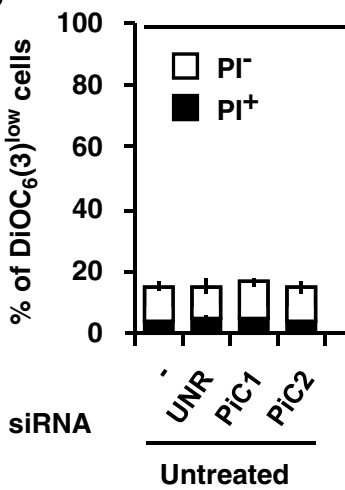

HeLa vMIA
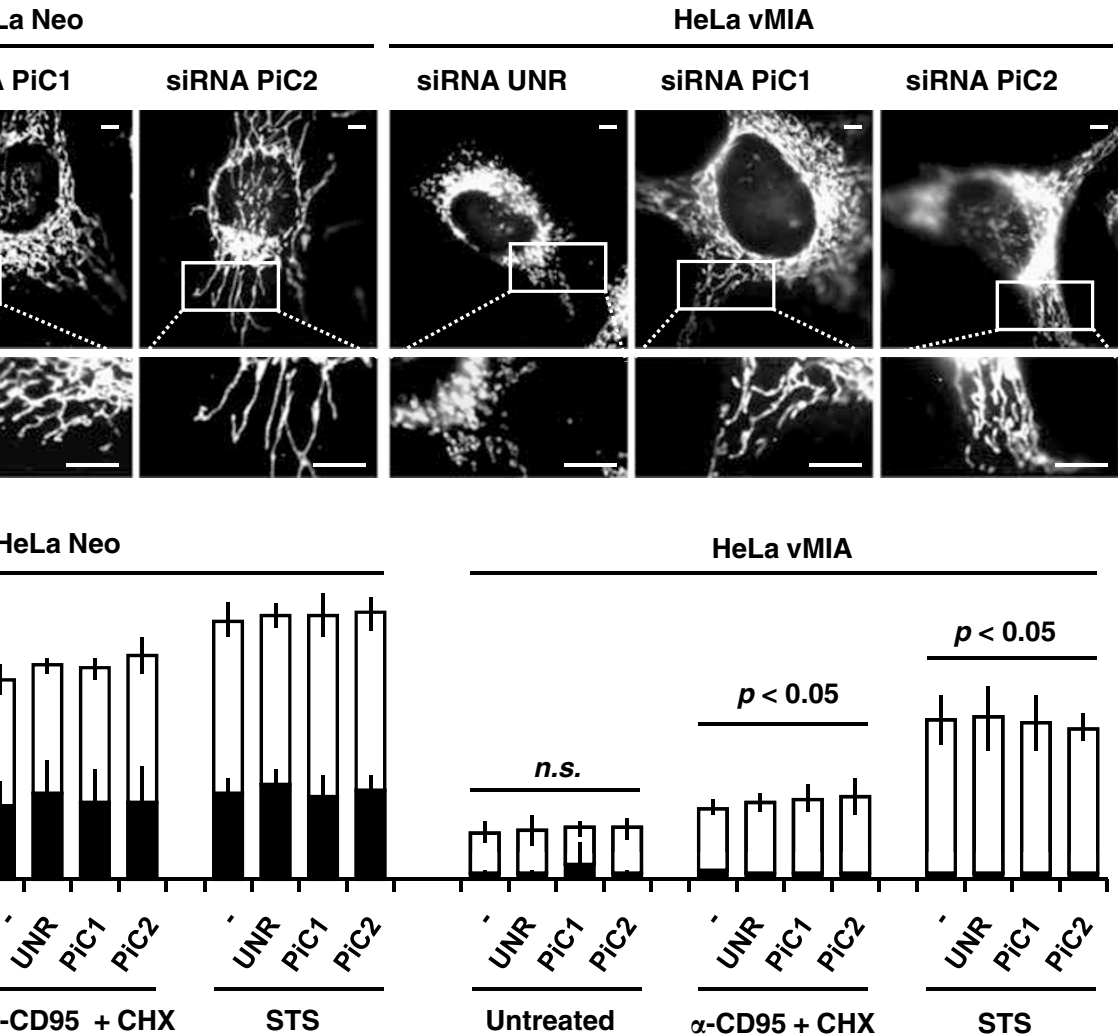

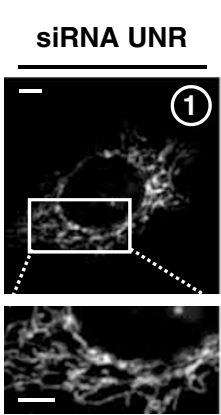

Normal

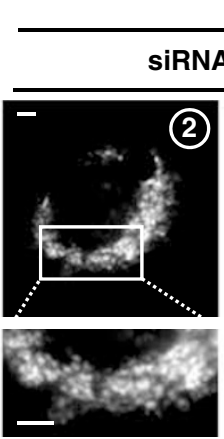

Microfragm.
siRNA Mfn-2

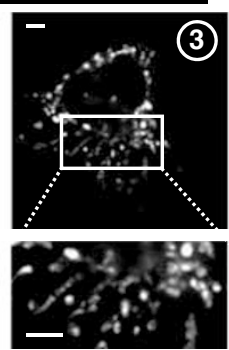

Macrofragm.
SIRNAPiC



Normal e

SIRNA UNR $\square$ siRNA PiC

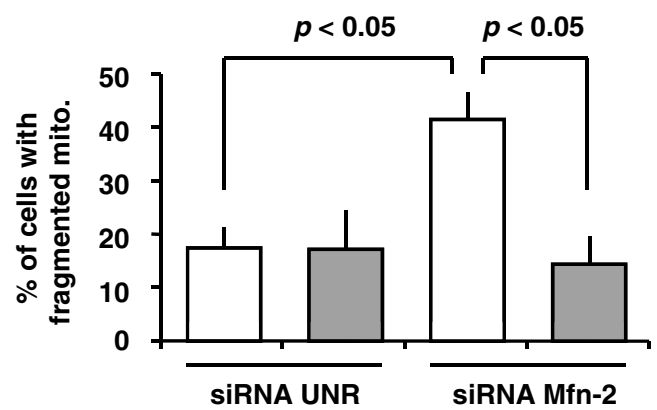


(a trigger for the extrinsic apoptotic pathway), even when $\mathrm{PiC}$ was depleted (Figure 1c). Hence, $\mathrm{PiC}$ is required for the mitochondrial fragmentation caused by VMIA, yet has no role in VMIA-mediated apoptosis inhibition. These data underline the notion that mitochondrial fragmentation and initiation of the mitochondrial pathway of apoptosis do not correlate in an obligatory fashion. ${ }^{13,15}$ This holds true also at a molecular level, as recently demonstrated for the protein hFis $1 .{ }^{16}$ Notably, hFis 1 (that normally promotes both apoptosis and mitochondrial fragmentation) loses selectively its lethal effects (not the ability to fragment mitochondria) in cells lacking the proapoptotic modulators Bax and Bak, or upon different mutations in the intermembrane region. ${ }^{16}$

Next, we investigated whether the contribution of $\mathrm{PiC}$ to mitochondrial fragmentation would be restricted to this particular system of fragmentation (i.e. that stimulated by VMIA) or whether PiC might play a general role in this process. Mitofusin 2 (Mfn-2) is a GTPase required for mitochondrial fusion the absence which has been previously shown to promote mitochondrial fragmentation. ${ }^{17}$ HeLa Neo cells transfected with an irrelevant siRNA exhibited a multibranched, reticular mitochondrial network (Figure 1d, panel 1). On the contrary, mitochondria from cells depleted for Mfn-2 appeared either with a finely punctuated perinuclear pattern (microfragmentation; Figure 1d, panel 2) or as large dots dispersed throughout the cytoplasm (macrofragmentation; Figure 1d, panel 3). Again, this effect was abolished by the simultaneous knockdown of PiC, which still failed to affect mitochondrial morphology per se (Figure 1d, panel 4; Figure 1e). The same effect was observed also in a nonsmall cell lung cancer cell line (A549), which exhibited only macro- (and not micro-) fragmented mitochondria following the knockdown of Mfn-2 (Supplementary Figure 1). Hence, $\mathrm{PiC}$ may play a rather general role in mitochondrial dynamics. At this point, the exact mechanism by which $\mathrm{PiC}$ participates in mitochondrial fragmentation is unknown. Although it appears plausible that mitochondrial fission requires $\mathrm{PiC}$ at some point, it remains to be established whether $\mathrm{PiC}$ directly contributes to the fission machinery at the inner mitochondrial membrane, or whether the metabolic alterations induced by $\mathrm{PiC}$ depletion (and a consequent phosphate deficiency within the mitochondrial matrix) indirectly impact on the equilibrium of fusion and fission events. Previous reports indicate that changes in energy substrate availability induce deep structural modifications of the mitochondrial network, aimed to accommodate increased amount of the respiratory chain components. ${ }^{18}$ In this regard, PiC downregulation might (at least theoretically) counteract mitochondrial fragmentation via a similar effect, yet it seems very improbable that the lack of PiC would favor, instead of inhibiting (by limiting the availability of inorganic phosphate for ATP synthesis), oxidative phosphorylation. ${ }^{18}$

Irrespective of these unresolved issues, it appears clear that $\mathrm{PiC}$ can profoundly influence mitochondrial dynamics. Although there is overwhelming correlative evidence, in several species, that mitochondrial fragmentation correlates with mitochondrial outer membrane permeabilization, ${ }^{19}$ the present report illustrates that this correlation is not absolute. Thus, the antiapoptotic protein VMIA induces mitochondrial fragmentation in a process that is clearly unrelated to its apoptosis-regulatory function.

Acknowledgements. GK is supported by Ligue contre le Cancer, Agence Nationale pour la Recherche (ANR), Cancéropôle lle-de-France, European Commission (Active p53, Apo-Sys, Chemores, TransDeath, Right, Death-Train) and Institut National du Cancer (INCa).

\section{A-L Pauleau 1,2,3,4,7, L Galluzzi 1,2,3,7, SR Scholz,5,6, $N$ Larochette $^{2,5}, \mathrm{O}$ Kepp $^{1,2}$ and G Kroemer Kr, 1,2,3 $^{*}$}

1 INSERM, U848, 39 rue Camille Desmoulins, Villejuif, France;

2 Institut Gustave Roussy, 39 rue Camille Desmoulins, Villejuif, France;

${ }^{3}$ Université Paris-Sud 11, 39 rue Camille Desmoulins, Villejuif, France;

${ }^{4}$ German Cancer Research Center (DKFZ), Redox Regulation (A160), Im Neuenheimer Feld 280, Heidelberg, Germany;

${ }^{5}$ CNRS, FRE2939, 39 rue Camille Desmoulins, Villejuif, France;

${ }^{6} \mathrm{ZMBH}$, Universität Heidelberg, Im Neuenheimer Feld 282, Heidelberg, Germany

7 A-L $P$ and $L G$ contributed equally to this paper.

* Corresponding author: G Kroemer, INSERM U848, Institut Gustave Roussy, PR1, 39 rue Camille Desmoulins, Villejuif F-94805, France.

Tel: + 3314211 6046; Fax: + 3314211 6047; E-mail: kroemer@igr.fr

1. Palmieri F. Pflugers Arch 2004; $447: 689-709$

2. Marzo I et al. Science 1998; 281: 2027-2031.

3. Belzacq AS et al. Biochimie 2002; 84: 167-176.

4. Le Bras M et al. Cancer Res 2006; 66: 9143-9152.

5. Kroemer G et al. Physiol Rev 2007; 87: 99-163.

6. Grinberg M et al. Mol Cell Biol 2005; 25: 4579-4590.

7. Alcala S et al. Oncogene 2007; doi: 10.1038/s.onc. 1210600.

8. Poncet D et al. J Cell Biol 2006; 174: 985-996.

9. Goldmacher VS et al. Proc Natl Acad Sci USA 1999; 96: 12536-12541.

10. Poncet D et al. J Biol Chem 2004; 279: 22605-22614.

11. Arnoult D et al. Proc Natl Acad Sci USA 2004; 101: 7988-7993.

12. Pauleau AL et al. Oncogene 2007; 26: 7067-7080.

13. Roumier T et al. Cell Death Differ 2006; 13: 348-351.

14. Perfettini JL, Roumier T, Kroemer G. Trends Cell Biol 2005; 15: 179-183.

15. Parone PA et al. Mol Cell Biol 2006; 26: 7397-7408.

16. Alirol E et al. Mol Biol Cell 2006; 17: 4593-4605.

17. Chen H et al. J Cell Biol 2003; 160: 189-200.

18. Rossignol R et al. Cancer Res 2004; 64: 985-993.

19. Karbowski M et al. Nature 2006; 443: 658-662. 